\title{
Calidad asistencial en la insuficiencia cardiaca
}

Muiño Míguez A. Calidad asistencial en la insuficiencia cardiaca. An Med Interna (Madrid) 2005; 22: 307-308.

La morbilidad y mortalidad que provoca la insuficiencia cardiaca sigue siendo elevada, a pesar de los avances en el conocimiento de su fisiopatología y tratamiento. Los pacientes presentan reingresos hospitalarios frecuentes con el consiguiente perjuicio para ellos y elevados costes para la sociedad, especialmente los ancianos.

Entre los esfuerzos para mejorar la calidad de la atención a los pacientes con insuficiencia cardiaca se pueden destacar la creación de guías de práctica clínica basadas en la evidencia científica, así como novedosas iniciativas organizativas para ofrecer una atención integral como son las unidades de insuficiencia cardiaca.

El escaso seguimiento de las guías podría ser, al menos en parte, una de las causas de la inaceptable morbimortalidad que sigue produciendo esta entidad (1). La evaluación del grado de aplicación de estas guías se realiza mediante estudios que utilizan indicadores de calidad.

Un indicador de calidad es la herramienta utilizada para medir la calidad en un aspecto clave de la atención. Hay dos tipos principales de indicadores: de estructura y de procesos o resultados. En estos últimos se analiza la actuación ante la enfermedad en la detección, evaluación inicial y diagnóstico, tratamiento, seguimiento, etc. En cada uno de éstos se consideran los aspectos más relevantes sobre la base de la evidencia científica y las guías de práctica clínica. No todos los indicadores tienen el mismo nivel de exigencia: a) básicos, de obligado cumplimiento; y b) óptimos, recomendables pero no obligados. La aplicación de indicadores adecuadamente seleccionados permite identificar áreas de mejora potencial, detectar problemas en el manejo clínico, incita a estudios de investigación en calidad y fomenta la cultura de la medida y la evaluación.

Álvarez y cols. (2) estudian en un Servicio de Medicina Interna el cumplimiento de los indicadores de calidad recogidos en el estudio ACOVE (3), alcanzando casi un $70 \%$ del ideal. Otros estudios como el ADHERE (4), estudio multicéntrico sobre 81.142 hospitalizaciones en 223 hospitales de Estados Unidos, evalúan cuatro indicadores de calidad asistencial recomendados por la Joint Commission on Accreditation of Healthcare Organizations: a) instrucciones médicas al alta con información sobre medicamentos, cita de seguimiento, signos a tener en cuenta y recomendaciones sobre qué hacer si se presentan complicaciones e información sobre los beneficio del ejercicio y una dieta baja en sodio; b) valoración de la función ventricular; c) uso de IECAs en pacientes con disfunción ventricular sistólica; y d) recomendaciones a los fumadores sobre el abandono del hábito. Además analiza la estancia media hospitalaria y mortalidad. El grado de cumplimiento registrado fue del $24,86,72$ y $43 \%$ respectivamente; la estancia media fue 4,5 días (3,3-9,5 días) y la mortalidad intrahospitalaria 4,2\% (0-11,1\%), con notables diferencias de unos hospitales a otros (4).

Una estrategia para mejorar la atención de estos pacientes que ha despertado un notable interés es el desarrollo en la última década de unidades especializadas en insuficiencia cardiaca (5-7). Estas ofrecen una atención continuada, en lugar de la organización tradicional basada en centros para la atención de las crisis o descompensaciones. En ellas se realizan diversas intervenciones como la actuación multidisciplinaria que permite una aproximación "holística" adaptada a cada paciente. Entre estas intervenciones se incluye la participación de personal de enfermería especialmente entrenado en el manejo ambulatorio, el registro de los datos relevantes del proceso asistencial como el uso de inhibidores de la ECA y betabloqueantes (fármacos que pese a haber demostrado su eficacia para aumentar la supervivencia son infrautilizados) (8), y rehabilitación cardiaca con educación del paciente, ejercicios y modificación del estilo de vida. Esta estrategia, ya ha demostrado su eficacia para mejorar la situación clínica, disminuir las hospitalizaciones, aumentar la calidad de vida y disminuir los costes (9-13).

Las mejoras en la calidad de la atención no dependen siempre de actuaciones costosas y complejas. Un estudio reciente en nuestro país ha demostrado que una sencilla intervención educativa como realizar una única visita por personal de enfermería en el domicilio del paciente una semana después del alta hospitalaria, consigue reducir el número de reingresos y visitas al servicio de urgencias con una mejora en la salud percibida (14). 
La atención a los pacientes con insuficiencia cardiaca constituye un importante reto que implica diversos niveles asistenciales y especialidades médicas, entre las que la medicina interna debe tener un papel destacado e integrador $(15,16)$. La atención de calidad requiere que la actuación sanitaria sea acorde con los conocimientos actuales y contribuya efectivamente a obtener el resultado deseado sobre la salud de los pacientes.

Estudios como el de Álvarez y cols. (2) son de gran utilidad para favorecer la implementación y adherencia a las directrices de buena práctica clínica y mejorar la calidad de la asistencia, lo que sin duda tendrá un importante impacto en la calidad de vida de los pacientes con insuficiencia cardiaca.

\section{A. MUIÑO MÍGUEZ}

Servicio de Medicina Interna II. Hospital General

Universitario Gregorio Marañón. Madrid

\section{Bibliografía}

1. Amin AN. The role of hospitalists in the management of acute decompensated heart failure.Am Heart Hosp J 2005 Spring; 3: 111-7.

2. Álvarez MT, Gutiérrez J, Alonso JL, Solano M, González C, Etxegaray $M$. Calidad del manejo de pacientes con insuficiencia cardiaca en el Servicio de Medicina Interna. An Med Interna (Madrid) 2005; 22: 309-312.

3. Gregg C, Fonarow M. Quality indicators of the management of heart failure in vulnerable elders. ACOVE. Ann Intern Med 2001; 135: 694702.

4. Fonarrow GC, Nancy CW, Heywood JT, ADHERE Scientific advisory comité, Study group, and Investigators. Adherence to heart failure quality-of-care indicators in US hospitals: analysis of the ADHERE Registry. Arch Intern Med 2005: 165: 1469-1477.

5. Taylor S, Bestall J, Cotter S, Falshaw M, Hood S, Parsons S, Wood L, Underwood M. Clinical service organisation for heart failure.Cochrane Database Syst Rev 2005 Apr 18

6. Martineau P, Frenette M, Blais L, Sauve C. Multidisciplinary outpatient congestive heart failure clinic: impact on hospital admissions and emergency room visits. Can J Cardiol 2004; 20: 1205-11.

7. Gustafsson F, Arnold JM. Heart failure clinics and outpatient management: a review of the evidence and call for quality assurance. Eur Heart J 2004; 25: 1596-1604

8. Luthi JC, Flanders WD, Pitts SR, Burnand B, McClellan WM. Outcomes and the quality of care for patients hospitalized with heart failure. Int J Qual Health Care 2004; 16 : 201-10.

9. Akosah KO, Schaper AM Haus LM, Mathiason MA, Barnhart SI,

McHugh VL. Improving outcomes in heart failure in the community: long-term survival benefit of a disease-management program. Chest 2005; 127:2042-8.

10. De Keulenaer GW, Brutsaert DL. Urgent need to reorganize heart failure management: from paradoxes to heart failure clinics. Acta Cardiol 2005; 60: 179-84.

11. Austin J, Williams R, Ross L, Moseley L, Hutchison S. Randomised controlled trial of cardiac rehabilitation in elderly patients with heart failure. Eur J Heart Fail 2005; 7: 411-7.

12. Pulignano G, Scherillo M, Del Sindaco D, Giulivi A, Giovannini E. Quality of care and management programs for the elderly with heart failure. Ital Heart J 2004; 5 (Supl. 10): 74S-86S.

13. Gwadry-Sridhar FH, Flintoft V, Lee DS, Lee H Guyatt GH. A systematic review and meta-analysis of studies comparing readmission rates and mortality rates in patients with heart failure. Arch Intern Med 2004; 164: 2315-20.

14. Morcillo C, Valderas JM, Aguado O, Delás J, Sort D, Pujadas R, Rosell F. Evaluación de una intervención domiciliaria en pacientes con insuficiencia cardiaca. Resultados de un estudio aleatorizado. Rev Esp Cardiol 2005; 58: 618-625.

15. Suárez P, Gabarró N, Gil J, Millán J. Evaluación de la calidad asistencial en la insuficiencia cardiaca. An Med Interna (Madrid) 1999; 16: 553-556.

16. Grupo de trabajo de insuficiencia cardiaca de la Sociedad Española de Medicina Interna. La insuficiencia cardiaca en los servicios de medicina interna (estudio SEMI-IC). Med Clin (Barc) 2002; 118: 605-610. 


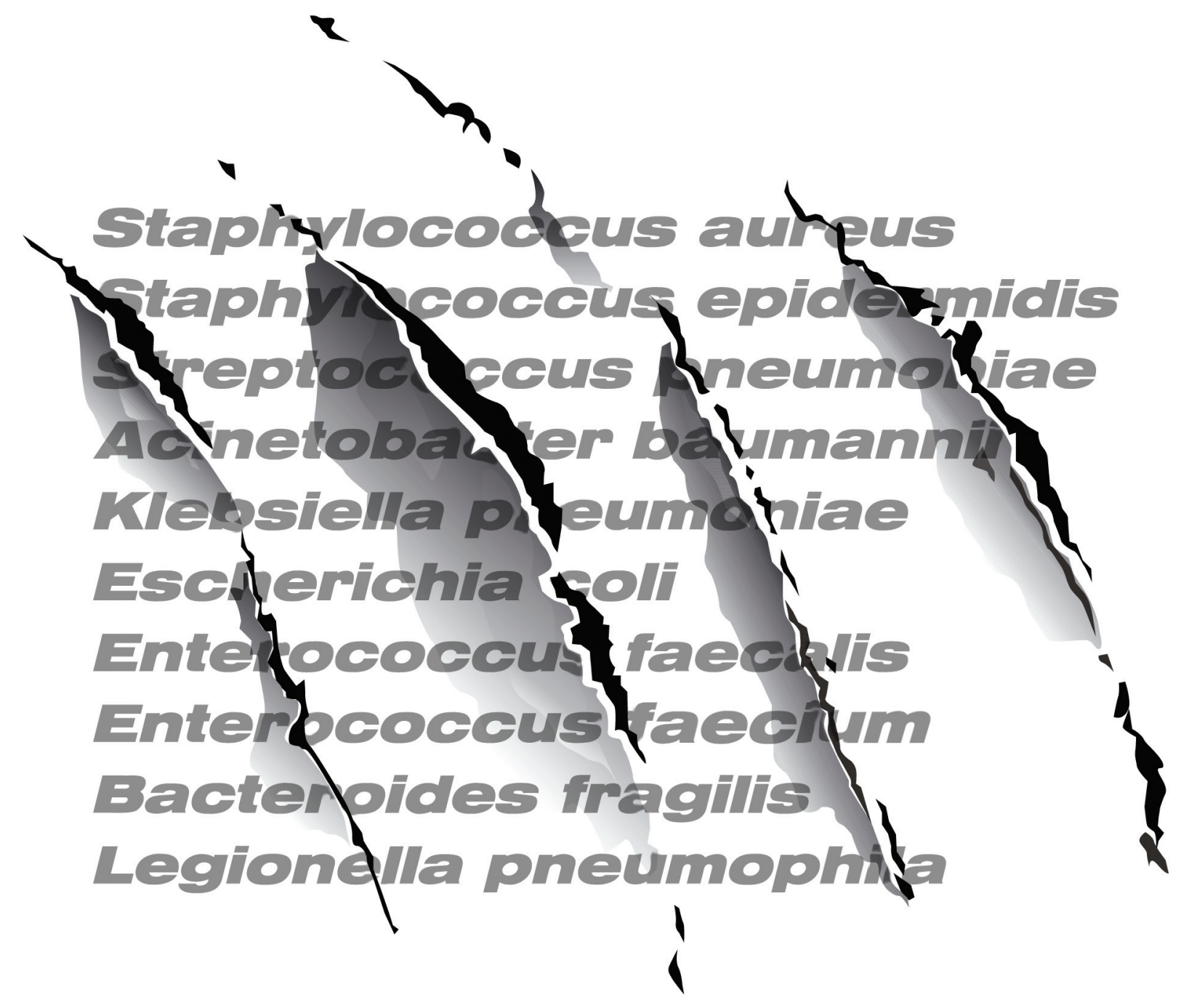

¿DE AMPLIO ESPECTRO O MULTIRRESISTENCIA? 


\section{XXXV}

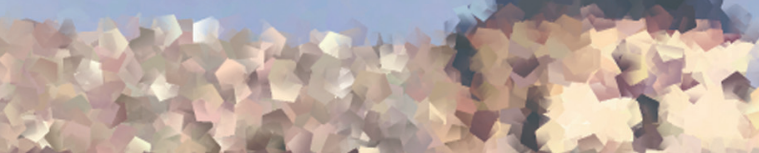
x.tate 13

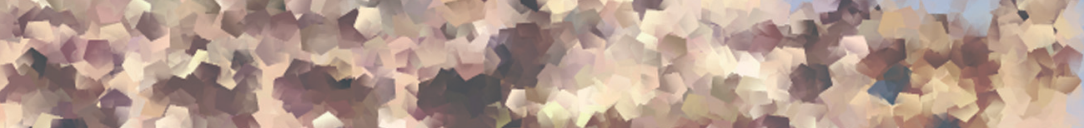

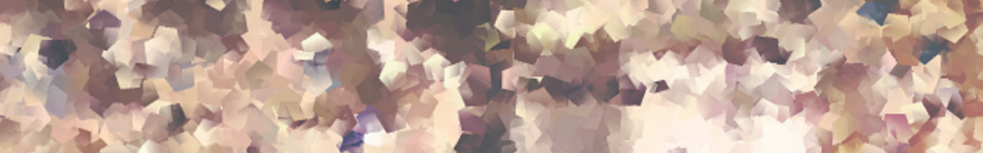
and $x+2$

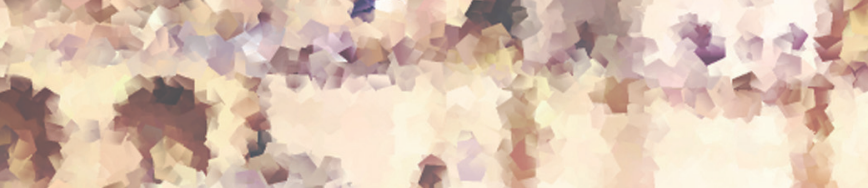
$\lim _{3}, 2$ II stat

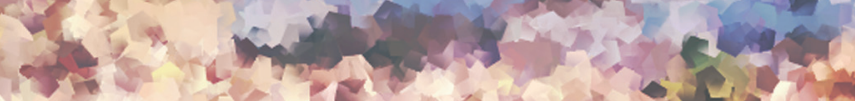

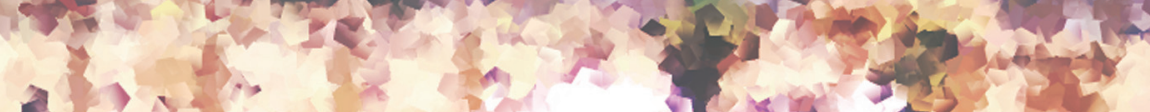

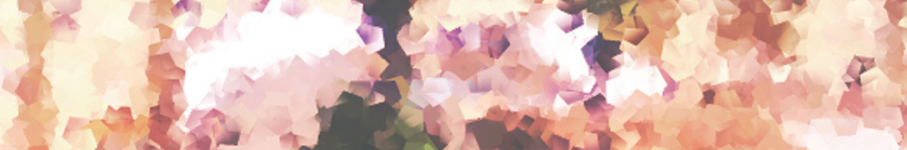
$2+20$ ath $c^{2}-1$

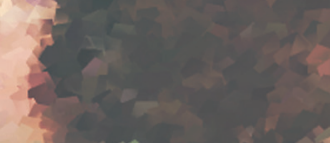

teste

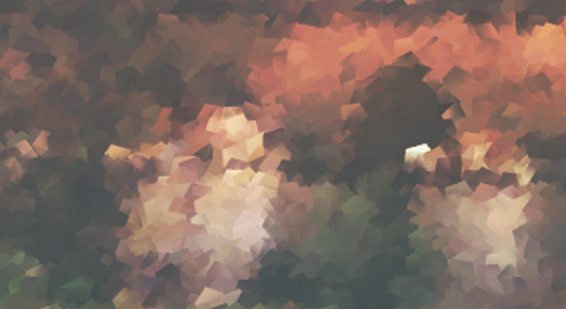
ximentis ers?

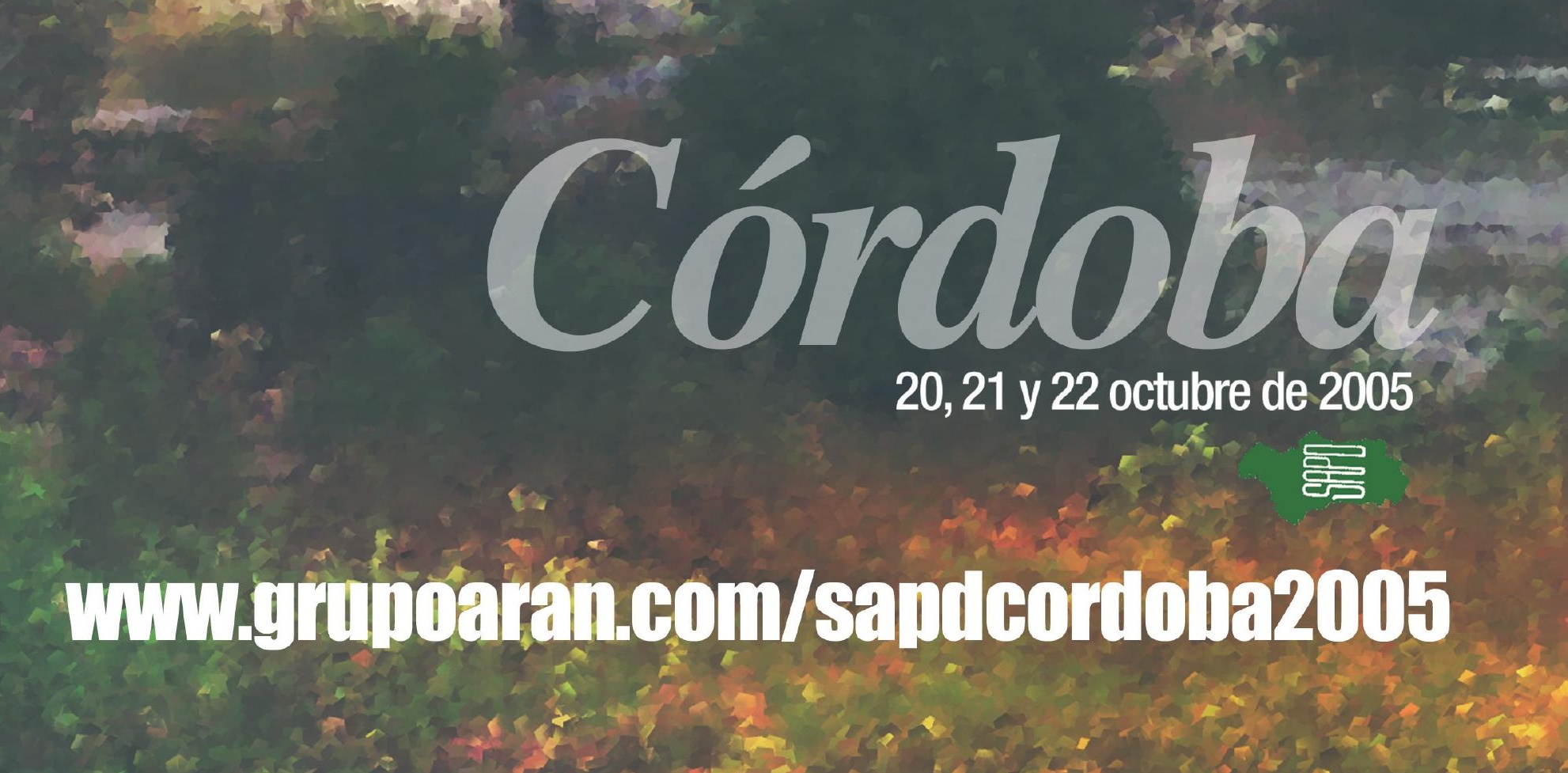

